\title{
Design of Nonlinear Flight Controller for Fighter Aircraft
}

\author{
P Lathasree*, Shaik Ismail**, \\ Abhay A Pashilkar* \\ *Scientist, Flight Mechanics \& Control Division, National Aerospace Laboratories, Bangalore, PB No. 1779 \\ India (Tel: 009180 25086533; e-mail:platha@nal.res.in, apash@nal.res.in). \\ ** Scientist (Retd), Flight Mechanics \& Control Division, National Aerospace Laboratories, \\ Bangalore, PB No.1779, India (e-mail: shaik1752@gmail.com)
}

\begin{abstract}
A nonlinear flight controller is developed using dynamic inversion principles. The nonlinearities in the equations of motion arising from inertia coupling and the gravity vector are compensated by dynamic inversion. Control and state decoupling is demonstrated for conventional aileron, elevator and rudder control surfaces using a static control allocation matrix and choice of stability axis rates for feedback respectively. We demonstrate that the right hand sides of the equations of motion can be approximated by using flight path variables and traditional feedback signals like normal and lateral accelerations. Further, except for the inertia compensation and gravity compensation terms which contain sine and cosine functions, the remainder of the controller can be designed in the linear domain. The simulation results are presented for a case where a nonlinear high performance fighter aircraft is undergoing a high angle of attack stability axis roll maneuver. This maneuver exercises the aircraft over a very wide dynamic range in a short time and demonstrates the capabilities of the nonlinear controller.
\end{abstract}

Keywords: Flight Control, Nonlinear model, dynamic inversion, control allocation, inertia coupling.

\section{INTRODUCTION}

Flight controller design is a well known inverse design problem and is compounded by the presence of nonlinearities in the equations of motion as well as the uncertain aerodynamic forces and moments. The nonlinear dependence of the aerodynamic forces and moments is not known exactly and the controller must be designed to be robust in the presence of plant parameter variations.

Gravity terms appear in the formulation of equation of motion as a function of aircraft's orientation in space. The kinematic and inertia cross coupling terms appear in equations of motion as products of motion variables. This coupling between motion variables cannot be neglected especially at high angles of attack and high rotational rates. When the aircraft rolls through 90deg about its body axis, the angle of attack gets converted to angle of sideslip (Harkegard 2001). This coupling is often referred to as kinematic coupling of longitudinal and lateral aircraft motions.

Simultaneous rotation about the two orthogonal axes will generate an angular acceleration along the direction perpendicular to both axes due to the gyroscopic moment. These angular acceleration components are due to inertial coupling and can be written as:

$$
\begin{aligned}
\Delta \dot{p} & =\left(\left(-I_{z}-I_{y}\right) / I_{x}\right) q r \\
\Delta \dot{q} & =\left(\left(-I_{x}-I_{z}\right) / I_{y}\right) p r \\
\Delta \dot{r} & =\left(\left(-I_{y}-I_{x}\right) / I_{z}\right) p q
\end{aligned}
$$

The current day high performance fighter aircraft are required to meet stringent performance criteria. Therefore it is required to address the issues arising from nonlinearities correctly. This is evident when the aircraft undergoes Herbst maneuver or J-turn (Li et al. 2001), as the nonlinearities are exhibited as a consequence of nonlinear aerodynamics, nonlinear inertial and kinematic couplings due to high angle of attack and high angular rates.

Nonlinear flight controller design is recommended for the aircraft which operate in the nonlinear regimes of flight where kinematic and inertia coupling terms and gravity terms dominate. The most widely used approach called Feedback Linearisation transforms a nonlinear system into a system exhibiting linear dynamics so that linear control methods can be applied to it. Nonlinear Dynamic Inversion (NDI), a specific case of feedback linearising control has been explored extensively for the control application of super maneuverable fighter aircraft. (Lane et al. 1988; Bugajski et al. 1992; Snell et al. 1992). Although NDI is an effective way of compensating nonlinearities associated with high angle of attack flight, it is very sensitive to uncertainties. The performance of the NDI controller deteriorates in the 
presence of unmodeled dynamics and parametric uncertainty, if the dynamic model of the aircraft not known exactly (Chao Zheng 2012). A variety of robust nonlinear control schemes have been proposed in literature that can be used in conjunction with NDI methodology.

Back Stepping techniques can also be used to control a nonlinear system that does not rely entirely on dynamic inversion (Krstic et al. 1995). Backstepping is a recursive, Lyapunov based nonlinear design method which could be applied to a nonlinear system in lower triangular form.

The known control approaches for the high angle of attack designs which address carefree maneuvering and boundary limiting are (Chien et al. 1990):

- Classical control designs with gain scheduling where the performance is satisfactory with low alpha regimes and may deteriorate with increasing roll rate (Snell et al. 1992)

- Nonlinear inverse dynamic technique was used to "invert" the nonlinear system such that system is decoupled and output can be individually controlled (Meyer and Cicolani 1981; Lane and Stengel 1988).

- Application of variable structure control to aircraft where the feedback gains were derived from Lyapunov-based equations and switched on hypersurfaces corresponding to different system dynamics (Singh 1989; Innocenti 1989).

- Block backstepping is a Lyapunov based technique for controller design and performs well with maneuvers such as high-alpha flight and high-rate velocity vector rolls (Robinson 2007).

- Bifurcation control to stabilize a high-alpha vehicle (Abed 1989)

- $\quad$ Thrust vectoring control (Albion and Joseph 1996; Stalford and Hoffman 1989)

- Control laws based on Differential PI algorithms (Osterhuber and Hanel 2004)

This paper discusses the development of NDI based controller using stability axis angular rates instead of the body axis angular rates and control decoupling using an optimally chosen fixed control allocation matrix. The major contribution of the paper is to demonstrate that the right hand sides of the equations of motion can be approximated by using flight path variables $(\mu, \chi, \gamma)$ and traditional feedback signals like normal and lateral accelerations. Further, except for the inertia compensation (which have product of body rates and moments of inertia) and gravity compensation terms (containing sine and cosine functions), the remainder of the controller can be designed in the linear domain. The choice of unified formulation for slow and fast states in wind axis and stability axis is a novel approach. The inner loop feedback uses the stability axis angular rates and the outer loop feedback uses the wind axis slow states $\alpha, \mu$ and $\beta$.
This paper demonstrates control decoupling with static control allocation matrix and an NDI controller. Simultaneous inner loop control decoupling and state decoupling is achieved about the stability axes by the use of the control allocation matrix. Kinematic coupling observed during the high alpha velocity vector rolls can be addressed with this controller. On the other hand, due to lack of control power, post stall maneuverability is not addressed.

At high angles of attack, the maximum allowable sideslip during a roll is in the order of 3-5 deg (Wayne et al. 1994) depending on the particular planform. To overcome kinematic coupling, we could roll about the wind axis (known as velocity vector roll). Then angle of attack and sideslip remain unchanged during a roll. With the assumption that a roll is performed at zero sideslip, this is equivalent to a stability-axis roll performed about the stability x-axis, $x_{s}$. In this case, the angular velocity $p_{s}$ is the variable to command. Similarly, when a sideslip is sought to be created for example during a crabbed landing, the stability axis yaw rate $\left(r_{s}\right)$ is the variable to command. Hence, the stability axis angular rates $\left(p_{s}\right.$ and $\left.r_{s}\right)$ are used instead of body axis angular rates for the feedback. To distribute the total control demand from a nonlinear controller among the available actuators, control allocation can be used (Harkegard 2003). The flight control designer can use control ganging, pseudo-inverse and optimization approaches to achieve control allocation.

The performance of the controller is evaluated with a high performance fighter aircraft subjected to high angle of attack stability axis roll maneuver (also referred to as velocity vector roll). This maneuver is similar to Herbst maneuver where there is a rapid change of direction of the flight path although we shall remain below stall. It is difficult to control this maneuver because the normal accelerations tends to draw heavy nose and tail portions of the aircraft farther from axis of rotation at high roll rate, resulting positive pitch rate and departure in the angle of attack.

The controller has been evaluated using a 6DOF nonlinear aircraft flight simulation package developed in Matlab / Simulink environment. The flight dynamic model was developed using the wind tunnel database. The actuators for each control surface are modeled as first order lags with a time constant of $0.05 \mathrm{sec}$. The rate limiter for each actuator is set to $60 \mathrm{deg} / \mathrm{s}$. The delays due to sensors and digital computation are represented by two samples delays in the closed loop feedback signals $(0.04 \mathrm{sec})$.

The rest of the paper is organized as follows. Section 2 discusses the unified model formulation in mixed axis system. The derivation of linear aircraft model with this formulation is explained along with the development of NDI controller with control decoupling using an optimally chosen fixed control allocation and using stability axis angular rates instead of the body axis angular rates. Section 3 presents the simulation results and Section 4 summarizes the conclusions from the study. 


\section{CONTROLLER DESIGN}

\subsection{Unified Model Formulation}

The 6 DOF equations of motion of rigid body fixed-wing aircraft are given in the form of twelve first order nonlinear differential equations. A mixed-axis system is used for simplifying the design of control laws based on dynamic inversion.

The rotational equations of motion are given in the body-axis system:

$$
\left[\begin{array}{c}
\dot{p} \\
\dot{q} \\
\dot{r}
\end{array}\right]=I^{-1}\left\{-\left[\begin{array}{ccc}
0 & -r & q \\
r & 0 & -p \\
-q & p & 0
\end{array}\right] I\left[\begin{array}{c}
p \\
q \\
r
\end{array}\right]+\left[\begin{array}{c}
l_{a} \\
m_{a} \\
n_{a}
\end{array}\right]+\left[\begin{array}{c}
l_{T} \\
m_{T} \\
n_{T}
\end{array}\right]\right\}
$$

where, $l_{a}, m_{a}, n_{a}$ are aerodynamic contributions, and $l_{T}$, $m_{T}, n_{T}$ are thrust contributions to pitch, yaw, and roll respectively. $I$ is the moment of inertia matrix. The states $(p, q, r)$ are 'fast states' because the control surface deflections have direct effect on their time derivatives $(\dot{p}, \dot{q}, \dot{r})$.

The body axis roll rate $(p)$ and yaw rate $(r)$ will be transformed into stability axis rates $\left(p_{s}, r_{s}\right)$ for the purpose of developing the control laws based on dynamic inversion:

$\left[\begin{array}{c}p_{s} \\ r_{s}\end{array}\right]=\left[\begin{array}{cc}\cos \alpha & \sin \alpha \\ -\sin \alpha & \cos \alpha\end{array}\right]\left[\begin{array}{l}p \\ r\end{array}\right]=T_{s}\left[\begin{array}{l}p \\ r\end{array}\right]$

Where, $\alpha$ is the angle of attack, and $T_{s}$ is the body-axis to stability-axis transformation matrix.

The wind-axis system is used to define dynamics of the 'slow states' - velocity roll angle $(\mu)$, angle-of-attack $(\alpha)$, and angle of sideslip $(\beta)$. Using the relationships developed in (Miele 1962), we can write

$$
\begin{aligned}
& \dot{\mu}=p_{s} \cos \beta+(q-\dot{\alpha}) \sin \beta+\dot{\chi} \sin \gamma \\
& \dot{\alpha}=q-p_{s} \tan \beta-\frac{(L-m g \cos \mu \cos \gamma)}{m V \cos \beta} \\
& \dot{\beta}=-r_{s}+\frac{g}{V} \sin \mu \cos \gamma-\frac{Y}{m V}
\end{aligned}
$$

where, $\chi$ is velocity yaw angle (heading angle) (Miele 1962), $\gamma$ is flight-path angle, $m$ is mass of aircraft, $g$ is acceleration due to gravity, $V$ is the velocity, $L$ is the lift, and $Y$ is the side-force. In flight control, the intent is to achieve decoupling between angle-of-attack and sideslip by rolling about the velocity vector. The variable $\mu$ correctly captures the angle of roll about the velocity vector. The state variables used in equations in our reformulation in (6-8) are not new; see for example (Bugakski 1992; Miele 1962; Reigelsperger et al. 1996). The novelty in our formulation lies in using the stability axis angular rates on the right hand side. This simplifies the control formulation as shown in the next section.

\subsection{Linear Aircraft Model}

A linear model of the high performance aircraft is chosen for capturing the dependence of the aerodynamics and engine moments. The linear model required for the design of the inner-most loop can be expressed in state-space form as:

$\dot{x}=A y+B u$

where,

$x=\left[\begin{array}{lll}q & p & r\end{array}\right]^{T}$

$y=\left[\begin{array}{lllll}\alpha & q & \beta & p & r\end{array}\right]^{T}$

$u=\left[\begin{array}{lllll}\delta_{e-l e f t} & \delta_{e-r i g h t} & \delta_{a-l e f t} & \delta_{a-r i g h t} & \delta_{r}\end{array}\right]^{T}$

and, $\delta_{e-l e f t}, \delta_{e-r i g h t}, \delta_{a-l e f t}, \delta_{a-r i g h t}$, and $\delta_{e-l e f t}, \delta_{r}$ are deflections of the left-elevator, right-elevator, leftaileron, right-aileron, and rudder respectively. A and $\mathrm{B}$ are plant and control matrices respectively. It is noted that we have deviated from the standard form of the linear equation in (12) by including the angle of attack and sideslip also into the state vector.

\subsection{Dynamic Inversion Control Law Design with the Unified Model Formulation}

Nonlinear dynamic inversion control method converts the affine nonlinear system to the pseudo linear system through the full state feedback and matrix inversion.

Therefore, the nonlinear dynamic inversion control law is designed by the linear control theory (Zhanqi and Li 2012). In conventional NDI control based on time scale separation, the design starts with the selection of a set of desired angular accelerations that allow the aircraft to complete a chosen maneuver.

Next, the body-axis rotational rates equations are solved with these desired angular rates using inversion of the complete nonlinear equations to get the required surface deflections. Similar procedure is used to design the loops for the slower states. As per the integrator backstepping concept, the angular rate demand for the inner loop is computed by the outer loop controller leading to a cascaded controller structure.

In this subsection we design a controller for the alpha command system for longitudinal axis, roll rate command system for lateral axis and sideslip/beta command system for directional axis respectively. The schematic of the NDI-based control law, developed in the present work, is shown in Figure 1. 


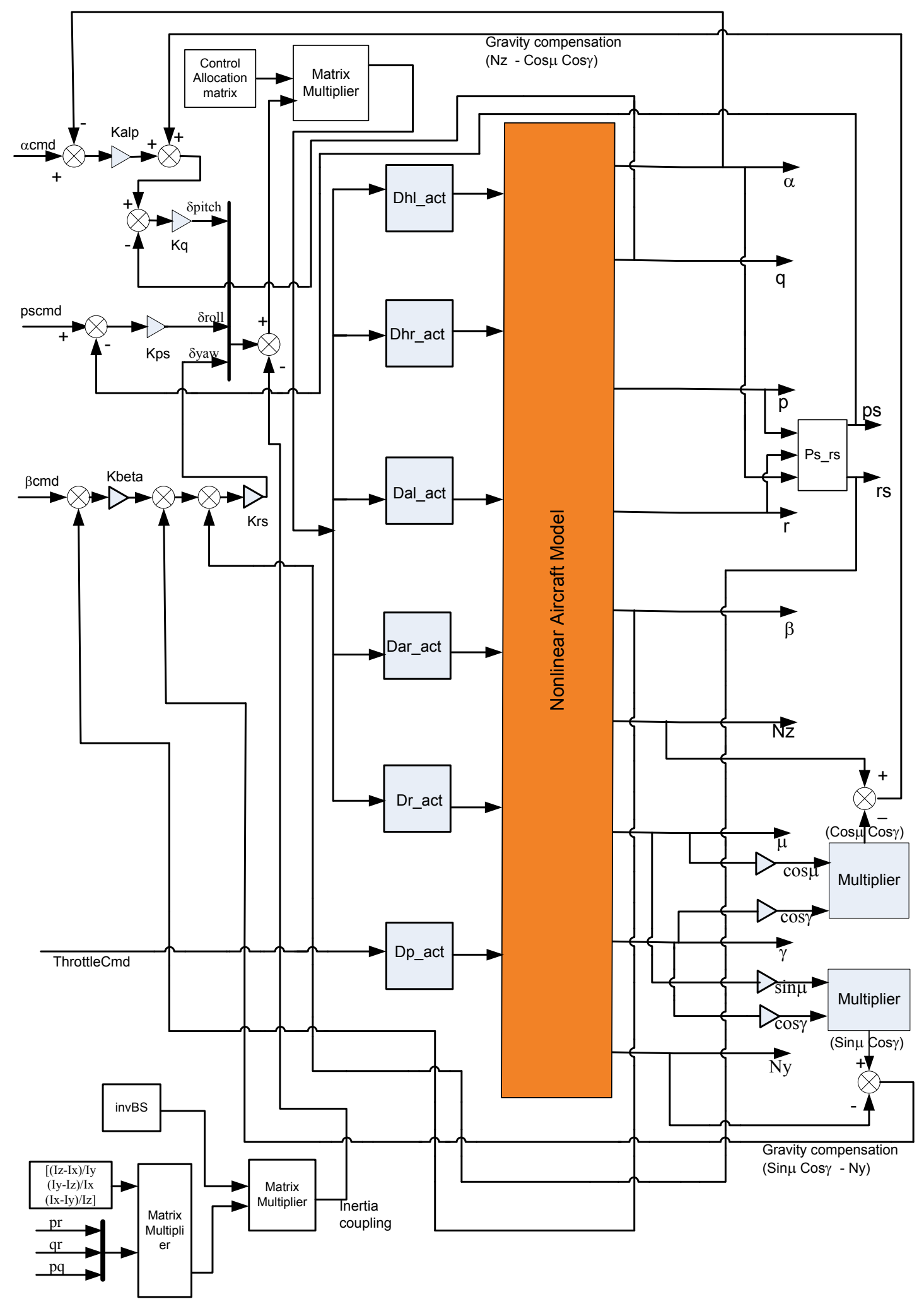

Figure 1 Block schematic of NDI controller 
Our controller is simplified compared to other results due to the use of stability axis rate feedback signals and by optimal choice of a fixed control allocation matrix. The cascade structure of the control law is the result of integrator backstepping. The design of the individual control loops are discussed in the following sections.

\subsubsection{Inner Loop Control Design $\left(p_{s}, q, r_{s}\right)$}

The innermost loop of the controller deals with the fast rotational dynamics. Under the assumption that the cross moments of inertia are negligible, the equations of rotational dynamics (4-6) can be rearranged as:

$$
\begin{aligned}
{\left[\begin{array}{c}
\dot{q} \\
\dot{p} \\
\dot{r}
\end{array}\right] } & =\left[\begin{array}{c}
\left(\left(I_{z}-I_{x}\right) / I_{y}\right) p r \\
\left(\left(I_{y}-I_{z}\right) / I_{x}\right) q r \\
\left(\left(I_{x}-I_{y}\right) / I_{z}\right) p q
\end{array}\right]+\left[\begin{array}{c}
\left(m_{a}+m_{T}\right) / I_{y} \\
\left(l_{a}+l_{T}\right) / I_{x} \\
\left(n_{a}+n_{T}\right) / I_{z}
\end{array}\right] \\
& =\left[\begin{array}{c}
\left(\left(I_{z}-I_{x}\right) / I_{y}\right) p r \\
\left(\left(I_{y}-I_{z}\right) / I_{x}\right) q r \\
\left(\left(I_{x}-I_{y}\right) / I_{z}\right) p q
\end{array}\right]+A y+B u
\end{aligned}
$$

where $I_{x}, I_{y}, I_{z}$ are the principle moments of inertia.

In the above equations we have retained the principle nonlinearities in the rotational equations in the form of the inertia coupling terms. The dependence of the aerodynamics and engine moments is approximated by the linear model explained in the previous section. We will employ a robust NDI based loop closure with suitable feedback structure for the aerodynamic terms to handle aerodynamic uncertainties. The design begins with decoupling of control surfaces, which appear in the rotational equations, by applying dynamic inversion to the above equations. For this purpose we define three pseudo-controls:

$\bar{u}=\left[\begin{array}{lll}\delta_{\text {pitch }} & \delta_{\text {roll }} & \delta_{\text {yaw }}\end{array}\right]^{T}$

These controls are used to exercise decoupled control of each of the three rotational axes. We also transform the roll rate and yaw rate into the stability axis rates. Thus, the transformed state and output vectors are given by:

$$
\begin{aligned}
& \bar{x}=\left[\begin{array}{lll}
q & p_{s} & r_{s}
\end{array}\right]^{T} \\
& \bar{y}=\left[\begin{array}{lllll}
\alpha & q & \beta & p_{s} & r_{s}
\end{array}\right]^{T}
\end{aligned}
$$

The matrices which transform the original variables $x, y$ and $u$ to their transformed quantities $\bar{x}, \bar{y}$ and $\bar{u}$ respectively are given by:

$$
\begin{aligned}
& x=\left[\begin{array}{c|c}
1 & 0_{1 \times 2} \\
\hline 0_{2 \times 1} & T_{s}^{-1}
\end{array}\right] \bar{x}=T_{1} \bar{x} \\
& y=\left[\begin{array}{c|c}
I_{3 \times 3} & 0_{3 \times 2} \\
\hline 0_{2 \times 3} & T_{s}^{-1}
\end{array}\right] \bar{y}=T_{2} \bar{y}
\end{aligned}
$$

$$
u=\left[\begin{array}{ccc}
1 & -\mathrm{K}_{\mathrm{aei}} & -\mathrm{K}_{\mathrm{rei}} \\
1 & \mathrm{~K}_{\mathrm{aei}} & \mathrm{K}_{\mathrm{rei}} \\
0 & -1 & 0 \\
0 & 1 & 0 \\
0 & \mathrm{~K}_{\mathrm{ari}} & 1
\end{array}\right] \bar{u}=S \bar{u}
$$

In (22), we have introduced two new gains $K_{a e i}$ and $K_{r e i}$ apart from the well known ailerons-to-rudder interconnect gain $K_{a r i}$. The ailerons-to-rudder interconnect suppresses the development of sideslip due to deflection of ailerons by application of proportional rudder. The gains $K_{a e i}$ and $K_{r e i}$ are intended to exploit the capability of the elevators in differential mode to generate additional rolling moment. The gain $K_{a e i}$ enables the controller to handle a new type of failure - the failure of both the ailerons simultaneously. The gain $K_{r e i}$ suppresses the development of rolling moments due to rudder by application of differential elevators and allows the controller to handle a larger range of rudder failures.

Using the above transformations, the linearized equations for rotational dynamics can be written as:

$T_{1} \dot{\bar{x}}=\left[\begin{array}{l}\left(\left(I_{z}-I_{x}\right) / I_{y}\right) p r \\ \left(\left(I_{y}-I_{z}\right) / I_{x}\right) q r \\ \left(\left(I_{x}-I_{y}\right) / I_{z}\right) p q\end{array}\right]+A T_{2} \bar{y}+B S \bar{u}$

Equation (23) can be rearranged as:

$$
\begin{aligned}
(B S)^{-1} T_{1} \dot{\bar{x}} & =(B S)^{-1}\left[\begin{array}{l}
\left(\left(I_{z}-I_{x}\right) / I_{y}\right) p r \\
\left(\left(I_{y}-I_{z}\right) / I_{x}\right) q r \\
\left(\left(I_{x}-I_{y}\right) / I_{z}\right) p q
\end{array}\right] \\
& +(B S)^{-1} A T_{2} \bar{y}+\bar{u}
\end{aligned}
$$

where $(B S)^{-1}$ is the inverse of the matrix product $(B S)$. If the diagonal entries of $(B S)$ do not change sign in the state space (i.e. there is no control reversal) then its inverse always exists. If the inverse does not exist, then it is not possible to obtain an acceptable control law and hence the system cannot be sufficiently controlled. For differentially non flat systems $(B S)^{-1}$ is not generally full rank, i.e. systems in which there are more states than control inputs, the number of states that can be inverted must be less than or equal to the number of control inputs available.

The gains $K_{a r i}, K_{a e i}$ and $K_{r e i}$ in the matrix $\mathrm{S}$ are chosen such that $(B S)^{-1} T_{1}$ is a diagonal matrix resulting in the control decoupling of pitch, roll and yaw axes .

Application of this to aircraft model at a suitable flight condition $(\mathrm{V}=150 \mathrm{~m} / \mathrm{s}$ and $\mathrm{h}=600 \mathrm{~m})$ with $K_{\text {ari }}=0.1 \mathrm{deg} / \mathrm{deg}$, $K_{a e i}=0.4 \mathrm{deg} / \mathrm{deg}$, and $K_{r e i}=0.31 \mathrm{deg} / \mathrm{deg}$ results in the longitudinal and lateral-directional decoupled equations. 


$$
\begin{aligned}
{\left[\begin{array}{c}
-5.05 \dot{q} \\
-1.28 \dot{p}_{s} \\
-13.55 \dot{r}_{s}
\end{array}\right] } & {\left[\begin{array}{c|ccc}
-1.68 & 0 & 0 \\
\hline 0 & -0.4278 & -0.028 \\
0 & 0.238 & -4.547
\end{array}\right]\left[\begin{array}{c}
0.96 p r \\
-0.77 q r \\
-0.73 p q
\end{array}\right] } \\
& +\left[\begin{array}{cc|ccc}
8.008 & 5.064 & 0 & 0 & 0 \\
\hline 0 & 0 & 34.33 & 4.18 & -0.95 \\
0 & 0 & -110.33 & -1.46 & 6.62
\end{array}\right]\left[\begin{array}{c}
\alpha \\
q \\
\beta \\
p_{s} \\
r_{s}
\end{array}\right] \\
& +\left[\begin{array}{c}
\delta_{\text {pitch }} \\
\delta_{\text {roll }} \\
\delta_{\text {yaw }}
\end{array}\right]
\end{aligned}
$$

The above equations (25) show the dependence of the rotational rates on the relevant aircraft states and the control inputs. The states $\alpha$ and $\beta$ are in degrees, and the rates are in $\mathrm{rad} / \mathrm{s}$.

Next, dynamic inversion control is designed to create a first order response in the variable being controlled. For, example the roll command is chosen to be:

$$
\begin{aligned}
\delta_{\text {roll }} & =-0.32 q r-0.0204 p q-34.33 \beta-4.183 p_{s} \\
& +0.9571 r_{s}+K_{p s}\left(p_{s c m d}-p_{s}\right)-1.28 \dot{p}_{s}
\end{aligned}
$$

where $p_{\text {scmd }}$ is the commanded roll rate input. Substitution of (26) into second equation in (25) will result in first order dynamics of the error state $e_{p s}=p_{s c m d}-p_{s}$. A value of $K_{p s}=-8.5 \mathrm{deg} / \mathrm{rad} / \mathrm{s}$ was chosen for the stability axis roll rate feedback gain. The value of $K_{p s}$ was chosen to ensure that the actuators do not rate limit during a high gain maneuver like the landing maneuver. It is noted that the actuators have a time constant of $0.05 \mathrm{~s}$. Then, the effective time constant of the first order response works out to be:

$\tau=\frac{-1.2861}{-8.5}=0.15 \mathrm{~s}$

Thus, the gains are chosen such that the time scale separation between the actuator bandwidth and the inner loop bandwidth is about three times. In the above roll control law (26), when the $K_{p s}$ gain is relatively high compared to the contribution from the second and fifth terms, they can be neglected. The third and fourth terms are stabilizing and therefore we do not cancel the same. Therefore we finally have the simplified roll control law.

$\delta_{\text {roll }} \cong-0.32 q r+K_{p s}\left(p_{\text {scmd }}-p_{s}\right)$

Similarly, the pitch and yaw axis inner loop control laws can be simplified as:

$$
\begin{aligned}
& \delta_{\text {pitch }} \cong-1.616 p r+K_{q}\left(q_{c m d}-q\right) \\
& \delta_{\text {yaw }} \cong 3.155 p q+K_{r s}\left(r_{s c m d}-r_{s}\right)
\end{aligned}
$$

It is desirable to have as high a gain in the inner loops (subject to not exciting actuator position and rate limits) from the point of view of handling actuator failures to compensate the disturbance moment created by the failure. The yaw gain is chosen to be $K_{r s}=-91 \mathrm{deg} / \mathrm{rad} / \mathrm{s}$ and $K_{q}=-33 \mathrm{deg} / \mathrm{rad} / \mathrm{s}$ resulting in the first order response time constant of $0.15 \mathrm{~s}$. It is noted that there is a time scale separation of about 3 times between the actuator bandwidth and the inner loop.

\subsubsection{Outer Loop Control Design $(\alpha, \beta)$}

For the outer loop control design, we assume that the dynamics of angle of attack $(\alpha)$, velocity roll angle $(\mu)$, and sideslip angle $(\beta)$ are slow compared to the states $\left(p_{s}, q, r_{s}\right)$. These assumptions lead to the following approximate dynamics:

$$
\begin{aligned}
& \dot{\alpha} \cong q-p_{s} \tan \beta-\frac{g}{V}\left(n_{z}-\cos \mu \cos \gamma\right) \\
& \dot{\beta} \cong-r_{s}+\frac{g}{V}\left(\sin \mu \cos \gamma-n_{y}\right)
\end{aligned}
$$

In the above equations, $n_{z}$ and $n_{y}$ are the lateral and normal accelerations respectively. It is noted that the measured signal $n_{z}$ is along the body $z$-axis and therefore is tilted by an angle equal to the angle of attack with respect to the lift force. Therefore as long as the range of operation is within about $30 \mathrm{deg}$ angle of attack this substitution is a reasonable approximation. The major advantage of using $n_{z}$ and $n_{y}$ is that it avoids the requirement of carrying an approximation of lift and side force within the nonlinear controller.

The outer loop dynamic inversion "control law" based on integrator backstepping concept applied to (31-32) is given by:

$$
\begin{aligned}
& q_{c m d}=K_{\alpha}\left(\alpha_{c m d}-\alpha\right)+\frac{g}{V}\left(n_{z}-\cos \mu \cos \gamma\right)+p_{s} \tan \beta \\
& r_{\text {scmd }}=-K_{\beta}\left(\beta_{r e f}-\beta\right)+\frac{g}{V}\left(\sin \mu \cos \gamma-n_{y}\right)
\end{aligned}
$$

The gains are chosen to be: $K_{\alpha}=2.5 \mathrm{rad} / \mathrm{s} / \mathrm{rad}$ and $K_{\beta}=0.5 \mathrm{rad} / \mathrm{s} / \mathrm{rad}$, resulting in the time constants of $0.4 \mathrm{~s}$ and 2.0 s respectively for these loops. It is to be noted that there is significant dynamic separation between the cascaded inner and outer loops.

The control inputs from the stick in pitch axis are scaled to demand an angle of attack. Similarly, the control inputs of the pilot in the roll channel create a demand for the stability axis roll rate. Finally, the rudder pedal inputs are scaled to create sideslip demand. The pilot operates the rudder, only to demand sideslip (e.g. during cross wind landing). 
The surface allocation strategy with control matrix inversion and coupling derivative compensation to decouple lateral and directional axes within full state feedback system are discussed in (Osterhuber and Hanel 2004). In contrast to (Osterhuber and Hanel 2004), our method of design for the inner loops achieves simultaneous state and control decoupling in the lateral-directional.

The control law structure considered for the lateraldirectional control is presented in Figure 1. It is to be noted that the gravity correction term is included to minimize the sideslip/beta. Figure 2 shows the responses of lateraldirectional parameters for the roll stick doublet input. It is evident from this figure that state decoupling is achieved with this controller. From the responses, it is observed that for the roll stick pulse input commands stability axis roll rate. The sideslip/beta response is small due to the introduction of gravity compensation.
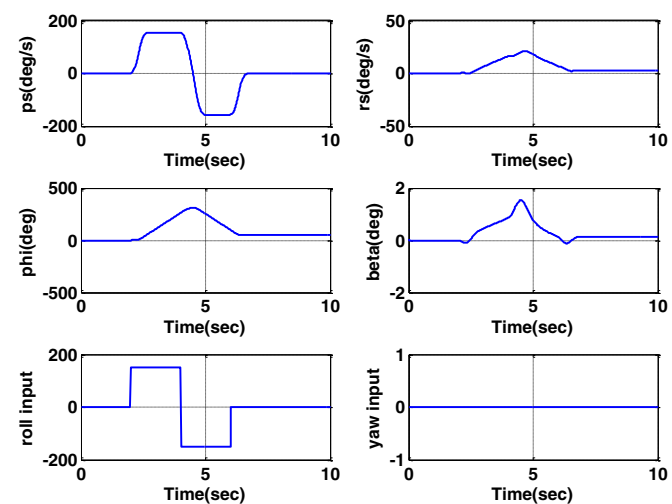

Figure 2 Linear responses for the roll pulse input

Table 1 Comparison of open loop and closed loop eigen values

\begin{tabular}{|c|c|c|c|}
\hline Mode & $\begin{array}{l}\text { Eigen } \\
\text { Values } \\
\text { Open Loop }\end{array}$ & $\begin{array}{l}\text { Eigen } \\
\text { Values } \\
\text { Closed Loop }\end{array}$ & $\begin{array}{l}\text { Handling } \\
\text { qualities } \\
\text { requirements }\end{array}$ \\
\hline $\begin{array}{l}\text { Roll } \\
\text { Mode }\end{array}$ & -3.2 & -2.7 & \\
\hline $\begin{array}{l}\text { Spiral } \\
\text { Mode }\end{array}$ & -0.0180 & -0.005 & \\
\hline $\begin{array}{l}\text { Dutch } \\
\text { Roll } \\
\text { Mode }\end{array}$ & $\begin{array}{ll}-0.3973 & + \\
2.7781 \mathrm{i} & \\
-0.3973 & - \\
2.7781 \mathrm{i} & \\
\zeta_{\text {dutchroll }} \\
0.142 \\
\omega \mathrm{n}_{\text {dutchroll }} \\
2.81 \mathrm{rad} / \mathrm{s}\end{array}$ & $\begin{array}{l}-11.2 \\
6.96 \mathrm{i} \\
-11.2-6.96 \mathrm{i} \\
\zeta_{\text {dutchroll }} \\
0.849 \\
\omega \mathrm{n}_{\text {dutchroll }} \\
13.2 \mathrm{rad} / \mathrm{s}\end{array}$ & $\begin{array}{l}\zeta_{\text {dutchroll }}>\mathbf{0 . 1 9} \\
\omega n_{\text {dutchroll }}>1.0 \\
\omega n_{\text {dutchroll }} \\
* \zeta_{\text {dutchroll }}>0.35\end{array}$ \\
\hline
\end{tabular}

The eigen values of the open loop plant and controller for the lateral - directional axes are presented below in the Table 1. From these values, it is noted that the open loop and closed loop system are stable. For the closed loop system, damping and natural frequency of dutch roll mode are meeting the handling quality requirements in contrast to the open loop system.

The responses of the lateral-directional parameters for multiple rolls using roll stick alone with and without gravity correction i.e. terms related to $(g / V)$ in $(33-34)$ are presented in Figure 3. It is seen that addition of these terms prevents sideslip response and angle of attack responses from diverging.
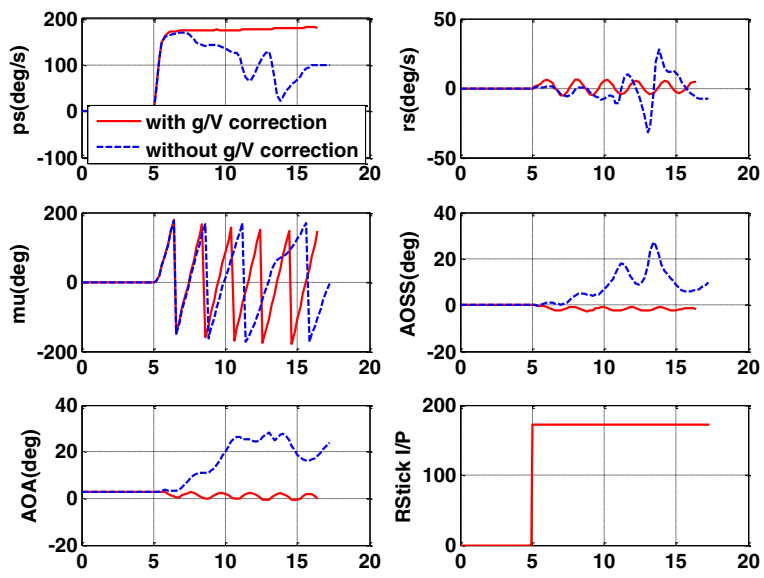

Figure 3 Nonlinear responses with multiple rolls with and without gravity compensation

\section{SIMULATION RESULTS}

To demonstrate the robustness in performance of NDI controller, simulation of Stability axis roll maneuver at high angles of attack is carried out. The maneuver starts with a straight and level flight at trim condition of $150 \mathrm{~m} / \mathrm{s}$, angle of attack $=2.8 \mathrm{deg}, \mathrm{h}=600 \mathrm{~m}$.

First the throttle is moved to its maximum deflection to cater for the expected loss of speed due to the high angle of attack excursion. A pitch command to the elevator is given to increase the angle of attack from its trim value at $3 \mathrm{sec}$. The command for the stability axis roll rate $\left(p_{s}\right)$ starts at $5 \mathrm{sec}$ and consists of three regions: the rising region (approximately $1 \mathrm{sec})$. The stability axis roll rotates the aircraft about its stability axis so as to turn the direction of the flight path vector. Then angle of attack is decreased to bring the nose down to the initial alpha at $18 \mathrm{sec}$.

The importance of developing NDI controller to control the above mentioned maneuver lies in the fact that this maneuver does exercise aircraft to wide dynamic range a short time and brings out the nonlinearities of the aircraft. Figure 4 shows the pitch stick and roll stick inputs for the high angle of attack roll axis maneuver along with the angle of attack, stability axis roll rate and sideslip responses. It is observed that the heading change at the end of the maneuver is about 180degrees. While the angle of attack is maintained as commanded, stability axis roll rate tends to reduce due to the loss of speed during the maneuver. The three dimensional trajectory (with pe, pn, h) is shown in the bottom right of the figure. 

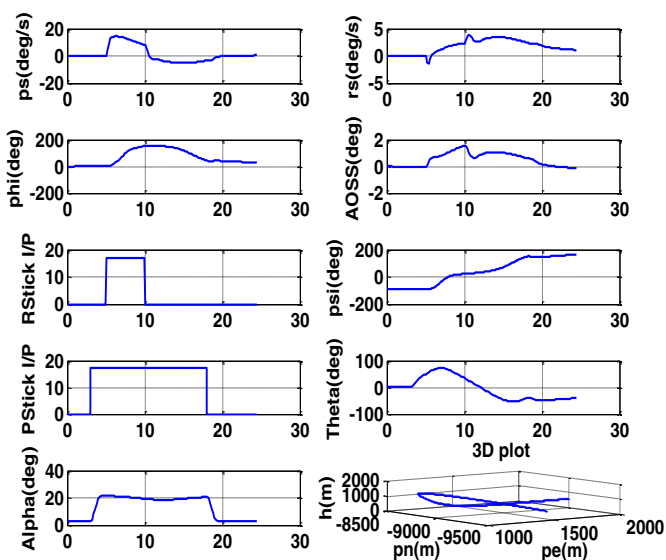

Figure 4 Responses for stability axis roll maneuver

\section{CONCLUSIONS}

A unified model formulation of the equations of motion has been derived in this paper and used for high performance controller design that is applicable to the class of fixed wing aircraft. A command (alpha, roll rate and sideslip/beta) following controller based on NDI approach is developed. It can be concluded that the NDI controller performs well for the tracking / command following tasks.

The design approach discussed in this paper has the following benefits: (1) Control and state decoupling of the innermost loops is achieved. (2) The loop gains are obtained in a natural manner based on the separation of time scales principles and literal expressions derived from the unified model formulation. This is in contrast to the classical loop shaping approach where the designer typically discovers the gain value by trial and error. (3) The command following controller discussed in this paper can be used for any fixedwing air vehicle including unmanned combat vehicles.

\section{REFERENCES}

Abed, Eyad H. (1989), Nonlinear Stabilizing Control of High Angle of Attack Flight Dynamics, AIAA 89-3487-CP

Albion H. Bowers and Joseph W. Pahle (1996), Thrust Vectoring on the NASA F-18 High Alpha Research Vehicle, NASA TM 4771

D. J. Bugajski, and D. F. Enns (1992), Nonlinear control law with application to high angle-of-attack, AIAA Journal of Guidance, Control, and Dynamics, 15, (3), pp. 761-767.

Chao Zheng (2012), Develop a robust nonlinear controller for large aircraft by applying NDI, SMC and adaptive control, M Sc Thesis, Cranfield University

Chien Y. Huang and Gareth J. Knowles (Decembr 1990), Application of Nonlinear Control Strategies to Aircraft at High Angle of Attack, Corporate Research Center, Grumman Corporation, MS A08-35, Bethpage, NY 11714, Proceedings of the 29th Conference on Decision and Control, Honolulu, Hawall

Fan Zhanqi, Liu Lin (2012), The high angle of attack aerodynamic modeling and nonlinear dynamic inversion flight control law design, 978-1-4673-0311-8/12, IEEE
O. Harkegard (2001), Flight Control design using back stepping, MS dissertation, Dept. Electrical Eng., Linkoping Univ., Linkoping, Sweden

O. Harkegard (2003), Backstepping and control allocation with applications to flight control, Ph.D. dissertation, Dept. Electrical Eng., Linkoping Univ., Linkoping, Sweden

Innocenti, Mario (1989), High Gain Flight Controllers for Nonlinear Systems, AIAA 89-3488-CP

John W. C. Robinson (2007), Block Backstepping for Nonlinear Flight Control Law Design, Nonlinear Analysis and Synthesis Techniques for Aircraft Control, Lecture Notes in Control and Information Sciences, 365, 231-257

Krstic M., Kanellakopoulos I. and Kokotovic P. V. (1995), Nonlinear and Adaptive Control Design, John Wiley \& Sons Inc. , New York, USA

Lane , S.H. and Stengel, R.F. (1988). Flight control design using nonlinear inverse dynamics, Automatica (Elsevier Journal), 24, (4), 471-483.

Y Li, N Sundararajan and P Saratchandran (2001), Neuro controller for nonlinear fighter aircraft maneuver using fully tuned RBF networks, Automatica (Elsevier Journal), 37, 1293-1301

Meyer, George and Cicolani, L. (1981), Applications of Nonlinear Systems Inverses to Automatic Flight Control Design - System Concepts and Flight Evaluations, AGARDOGRAPH 251 on Theory and Applications of Optimal Control in Aerospace Systems, NATO

Miele (1962), Flight Mechanics Volume I: Theory of Flight Paths, Addison-Wesley Pub. Co. Inc.

Robert Osterhuber and Dr M Hanel (2004), Realization of the Eurofighter 2000 Primary Lateral/Directional Flight Control Laws with Differential PI Algorithm, AIAA 2004-4751, Guidance, Navigation and Control Conference and Exhibit, Rhode Island

Singh, Sahjendra N. (1989), Asymptotically Decoupled Discontinuous Control of Systems and Nonlinear Aircraft Maneuver, IEEE Trans. on Aerospace and Electronic Systems, 25, (3), 380- 391

Snell, S.A., Enns, D.F. and Garrard W.L. Jr, (1992), Nonlinear inversion control for a supermaneuverable aircraft, AIAA Journal of Guidance, Control, and Dynamics, 15, (4), 976-984.

Stalford, Harold, and Hoffman Eric (1989), Thrust Vectoring Effect on Time-Optimal 90 Degrees Angle of Attack Pitch-up Maneuvers of a High-Alpha Fighter Aircraft, AIAA 89-3521 -CP

Wayne C. Durham, Frederick H. Lutze, and William Mason (1994), Kinematics and aerodynamics of the velocity vector roll, AIAA Journal of Guidance, Control, and Dynamics, 17(6):1228-1233.

W. C. Reigelsperger, S. S. Banda, and D. P. Lemaster (1996), Application of multivariable control theory to aircraft control laws. Final Report - Multivariable control design guidelines, WL-TR-96-3099, Flight Dynamics Directorate, Wright Laboratory, Wright-Patterson AFB, $\mathrm{OH}$. 